\title{
Body Mass Index, Metabolic Factors, and Striatal Activation During Stressful and Neutral-Relaxing States: An fMRI Study
}

\author{
Ania M Jastreboff', Marc N Potenza ${ }^{2,3}$, Cheryl Lacadie ${ }^{4}$, Kwangik A Hong ${ }^{2}$, Robert S Sherwin' \\ and Rajita Sinha*,2,3 \\ 'Department of Internal Medicine, Section of Endocrinology, Yale University School of Medicine, New Haven, CT, USA; ${ }^{2}$ Department of \\ Psychiatry, Yale University School of Medicine, New Haven, CT, USA; ${ }^{3}$ Child Study Center, Yale University School of Medicine, New Haven, \\ CT, USA; ${ }^{4}$ Department of Diagnostic Radiology, Yale University School of Medicine, New Haven, CT, USA
}

\begin{abstract}
Stress is associated with alterations in neural motivational-reward pathways in the ventral striatum (VS), hormonal/metabolic changes, and weight increases. The relationship between these different factors is not well understood. We hypothesized that body mass index (BMI) status and hormonal/metabolic factors would be associated with VS activation. We used functional magnetic resonance imaging ( $\mathrm{FMRI}$ ) to compare brain responses of overweight and obese (OW/OB: BMI $\geqslant 25 \mathrm{~kg} / \mathrm{m}^{2}: \mathrm{N}=27$ ) individuals with normal weight (NW: BMl $<18.5-24.9 \mathrm{~kg} / \mathrm{m}^{2}: \mathrm{N}=2 \mathrm{l}$ ) individuals during exposure to personalized stress, alcohol cue, and neutral-relaxing situations using a validated, autobiographical, script-driven, guided-imagery paradigm. Metabolic factors, including fasting plasma glucose (FPG), insulin, and leptin, were examined for their association with VS activation. Consistent with previous studies, stress and alcohol cue exposure each increased activity in cortico-limbic regions. Compared with NW individuals, OW/OB individuals showed greater VS activation in the neutral-relaxing and stress conditions. FPG was correlated with VS activation. Significant associations between VS activation and metabolic factors during stress and relaxation suggest the involvement of metabolic factors in striatal dysfunction in OW/ $\mathrm{OB}$ individuals. This relationship may contribute to non-homeostatic feeding in obesity.

Neuropsychopharmacology (20II) 36, 627-637; doi:I0.1038/npp.2010.194; published online 3 November 2010
\end{abstract}

Keywords: obesity; stress; ventral striatum; $\mathrm{PMRI}$; BMl; metabolic factors

\section{INTRODUCTION}

Obesity represents a significant public health concern. Over $68 \%$ of adults in the United States are overweight (OW) or obese (OB) with a body mass index (BMI) of $25-29.9 \mathrm{~kg} / \mathrm{m}^{2}$ or $\geqslant 30 \mathrm{~kg} / \mathrm{m}^{2}$, respectively (Flegal et al, 2010). With most of the US population above the recommended normal weight (NW) BMI $\left(18.5-24.9 \mathrm{~kg} / \mathrm{m}^{2}\right)$, many people develop obesityrelated conditions, including cardiovascular disease, type 2 diabetes, and various cancers (Ogden et al, 2007). It is estimated that obesity increases health care cost by $\$ 51.5-$ $\$ 78.5$ billion dollars annually (Finkelstein et al, 2003) and substantially impacts quality of life. Despite various treatment approaches, rates of obesity continue to escalate; therefore, novel prevention and treatment strategies targeting the causes of obesity are needed.

\footnotetext{
*Correspondence: Professor R Sinha, Department of Psychiatry, Yale Stress Center (yalestress.org), Yale University School of Medicine, Suite 209, 2 Church Street South, New Haven, CT 065 19, USA, Tel: + 203 737 5805, Fax: + 203737 1272, E-mail: rajita.sinha@yale.edu Received 26 June 2010; revised 17 September 2010; accepted 24 September 2010
}

The development of obesity is thought to involve multiple factors, including readily available, relatively inexpensive, highly caloric, and palatable foods; consumption of larger portions of food; a decrease in physical exertion needed to obtain food; and a predominantly sedentary lifestyle (Hill and Peters, 1998; Wang et al, 2004; Ogden et al, 2007). Genetic and other biological factors also contribute, making certain individuals more susceptible to gain weight in the context of these and other environmental factors (Stice $e t a l$, 2008; von Deneen et al, 2009). Behavioral aspects of eating may also contribute to weight status and weight changes. In particular, stress may influence eating behaviors and has been associated with increased weight (Adam and Epel, 2007; Block, 2009) and disordered eating (Dallman et al, 2003) in vulnerable individuals. It has also been associated with consumption of high-fat, calorie-dense foods, often to improve mood, possibly though reduction in central levels of corticotropin-releasing factor (Dallman et al, 2003). In the Midlife in the United States longitudinal study, individuals who were in an elevated BMI category at the onset of the study showed a stronger association between stress and future weight gain than their peers who started in the lean BMI group at baseline and experienced a comparable amount of stress (Block, 2009). This finding 
suggests that obesity may confer specific vulnerability to stress and stress-related food consumption and subsequent weight gain. In order to target stress as a potential modifiable factor in the development of obesity, it is important to examine its relation to BMI and clarify how stress influences eating behaviors and, subsequently, how stress reduction may improve or create potentially new and effective strategies for obesity treatment.

Insight into the association between obesity and stress may be gained from research in the addiction field as parallels may be drawn between overeating and excessive alcohol and drug use (Wang et al, 2004, 2009; Volkow and Wise, 2005; Frascella et al, 2010). Similar to alcohol and other drugs, highly caloric and palatable foods activate brain stress and motivational pathways that likely evolved to respond and adapt to challenging environments and primary rewards necessary for survival (Kelley and Berridge, 2002; Sinha, 2008). Chronic substance use (Sinha, 2008) and high BMI states are associated with alterations in stress pathways (Dallman et al, 2003) which in turn are associated with stress-related consummatory behaviors (Adam and Epel, 2007). A central component of the stress/reward motivational neurocircuitry involves the ventral striatum (VS), a structure implicated in reward processing (including reward-based learning and expectation, valuation, or anticipation of rewards) and stress responsiveness (Volkow et al, 2008). Stress, food, and drugs all increase neurotransmission in the VS (Pruessner et al, 2004; Kelley et al, 2005; von Deneen et al, 2009). A reduction in striatal dopamine 2/3 (DA D2) receptors in obese individuals similar in magnitude to those observed in drug-addicted individuals has been reported (Wang et al, 2001), suggesting alterations in striatal function in obese individuals in stress/reward neurocircuitry. Furthermore, DA D2 receptor measures correlate inversely with BMI, suggesting that individuals with the lowest levels of striatal DA D2 receptors have the highest BMIs (Wang et al, 2001). Together, these data suggest that similar aspects of striatal dysfunction may contribute to drug addiction, obesity, and stress vulnerability.

We hypothesized that individuals with elevated BMIs (OW/OB vs NW subjects) would exhibit altered VS activation during: (1) exposure to stressful stimuli and (2) induction of relaxed states. As the ability to relax may be impaired in individuals with an abnormal stress response, we hypothesized that the neural response to both stress and relaxing cues would be altered in OW/OB individuals.

To investigate, we employed a previously validated, individualized guided imagery script paradigm during functional magnetic resonance imaging (fMRI) to examine brain responses during stress, reward-appetitive (alcohol cue), and neutral-relaxing conditions in OW/OB individuals as compared with their NW counterparts. Subjects were light drinkers (less than 7 drinks per week) and not selected for differences in alcohol consumption, therefore no specific hypotheses were made with respect to the alcohol cue condition. As recent research has underscored the role of adipose signals, such as leptin and insulin, on central nervous system control of energy homeostasis (Woods and Seeley, 2000), we also assessed whether these and other metabolic factors related to BMI and obesity were associated with VS activity.

\section{METHODS}

\section{Subjects}

Healthy men and women aged 19-50 years, with BMI ranging from 18.5 to $36.6 \mathrm{~kg} / \mathrm{m}^{2}$, were recruited through local advertisements. Social drinking healthy control subjects from a previously published alcohol abuse investigation were studied, with social drinking defined as consuming less than seven drinks per week (Sinha et al, 2009). Participants were excluded if they reported any chronic medical conditions, were taking medications for medical problems or psychiatric disorders, met DSM-IV criteria for psychiatric disorders, or reported claustrophobia or significant metal in their body. All participants completed demographic and stress assessments and a Structured Clinical Interview (SCID-I) for DSM-IV (First et al, 1995). The study was approved by the Yale Human Investigation Committee. All subjects provided signed informed consent.

\section{Biochemical Evaluation}

Fasting blood samples were obtained at 0815 hours, immediately placed on ice and spun, and the plasma was stored at $-80^{\circ} \mathrm{C}$. Fasting plasma glucose (FPG) levels were measured using Delta Scientific glucose reagent (Henry Schein). Insulin and leptin levels were measured with a double antibody radioimmunoassay (Millipore, previously Linco). Each sample was analyzed in duplicate for verification. Neuroimaging was conducted within seven days of completion of laboratory data acquisition.

\section{Imagery Script Development}

Before fMRI, guided imagery scripts for stress, alcohol cues, and neutral-relaxing states were developed using previously established methods (see Sinha et al, 2009 for review). Stress scripts were developed from subjects' descriptions of two recent personal stressful events that were reported to be 'most stressful'. Individual calibration of 'most stressful' was determined by having the subjects rate their individual level of distress for each stressful situation on a 10-point Likert scale on which ' $1=$ not at all stressful' and ' $10=$ the most stress they felt recently in their life'. Only situations rated as 8 or above were accepted as appropriate for script development. Examples included a breakup with a significant other, unemployment, or death of a loved one. Alcohol cue scripts, representing an active control appetitive condition, were based on individual situations that included alcohol-related stimuli and resulted in alcohol consumption. An alcohol cue condition is an appetitive cue that for the group of social drinkers was anticipated to be approximately equally relevant across BMI groups, thus providing a comparison condition for the stress cues. Examples of alcohol script topics included meeting a friend at a bar and birthday celebrations. Alcohol-related situations that occurred in the context of negative affect or psychological distress were not used. Two neutral-relaxing scripts were developed from individual experiences of neutral-relaxing situations, such as a summer day relaxing at the beach or a fall day reading at the park. Sample scripts are provided (Supplementary Table 1). Script generation 
employed the Scene Construction Questionnaires (Miller et al, 1987; Sinha et al, 1992, 2003, 2004, 2005; Sinha, 2009). Each script was $2 \mathrm{~min}$ in duration, audio-recorded, and presented in randomized, counter-balanced order with six trials presented using a block design with one script per trial (two trials each of stress, neutral-relaxing, and alcoholrelated cue) during the fMRI session. The experimenters and research staff involved in data collection were blinded to the order and the content of the script stimuli in the fMRI session. Subjects remained blind to the order until presentation during imaging.

\section{Habituation and Imagery Training Session}

Before fMRI, subjects were acclimated to study procedures, such as the verbal analog scale for stress-related anxiety ratings, and trained in progressive relaxation and guided imagery procedures used during the fMRI session (Sinha, 2001, 2009).

\section{fMRI Acquisition}

Images were obtained using a 3-T Siemens Trio MRI system equipped with a standard quadrature head coil, using T2*-sensitive gradient-recalled single shot echo planar pulse sequence. Subjects were positioned in the coil and head movements were restrained using foam pillows. Anatomical images of the functional slice locations were obtained next with spin echo imaging in the axial plane parallel to the AC-PC line with $\mathrm{TR}=300 \mathrm{~ms}$, echo time $=2.5 \mathrm{~ms}$, bandwidth $=300 \mathrm{~Hz} /$ pixel, flip angle $=60$ degrees, field of view $=220 \times 220 \mathrm{~mm}$, matrix $=256 \times 256,32$ slices with slice thickness $=4 \mathrm{~mm}$, and no gap. Functional, blood oxygen level dependent signals were then acquired with a single-shot gradient echo planar imaging sequence. A total of 32 axial slices parallel to the AC-PC line covering the whole brain were acquired with $\mathrm{TR}=2000 \mathrm{~ms}$, echo time$25 \mathrm{~ms}$, bandwidth $=2005 \mathrm{~Hz} /$ pixel, flip angle $=85$ degrees, field of view $=220 \times 220 \mathrm{~mm}$, matrix $=64 \times 64$, 32 slices with slice thickness $=4 \mathrm{~mm}$, and no gap 190 measurements. Following fMRI, a high-resolution 3D Magnetization Prepared Rapid Gradient Echo sequence $(\mathrm{TR}=2530 \mathrm{~ms}$; echo time $=3.66 \mathrm{~ms} ;$ bandwidth $=180 \mathrm{~Hz} /$ pixel; flip angle $=7$ degrees; slice thickness $=1 \mathrm{~mm}$; field of view $=256 \times$ $256 \mathrm{~mm}$; matrix $=256 \times 256$ ) was used to acquire sagittal images for multi-subject registration.

\section{fMRI Trials}

Study participants underwent a $1.5 \mathrm{~h}$ fMRI session in which they were exposed in a randomized, counter-balanced fashion to two trials each of personalized stress, alcoholcue, and neutral-relaxing situations. Six fMRI trials (two per condition) were acquired using a block design with each lasting $5.5 \mathrm{~min}$. Each trial included a 1.5 -min quiet baseline period followed by a 2.5 -min imagery period (that included 2 min of read-imagery and $0.5 \mathrm{~min}$ of quiet-imagery) and a 1-min quiet recovery period. As previously (Sinha et al, 2004, 2005; Li et al, 2005), participants were requested to rate how tense, anxious and/or jittery they felt at that moment using a similar 10 -point verbal analog scale anchored as above before and after each fMRI trial. After each trial, subjects participated in progressive relaxation for 2-min periods to return anxiety and physiological state from the previous trial to baseline levels.

\section{fMRI Analysis}

All data were converted from Digital Imaging and Communication in Medicine format to analyze format using XMedCon (Nolfe et al, 2003). During conversion, the first 10 images at the beginning of each of the six functional series were discarded to enable the signal to achieve steadystate equilibrium, leaving 180 measurements for analysis. Images were motion corrected for three translational and three rotational directions using SPM5 (http://www.fil.ion. ucl.ac.uk/spm/software/spm5). Trials with linear motion in excess of $1.5 \mathrm{~mm}$ or rotation greater than 2 degrees were discarded. Individual subject data were analyzed using a General Linear Model on each voxel in the entire brain volume with a regressor specific for the baseline period and a second regressor for the imagery period for each trial. The resulting functional images for each script type were spatially smoothed with a 6-mm Gaussian kernel to account for variations in the location of activation across subjects. The output included normalized $\beta$-maps in the acquired space $(3.44 \times 3.44 \times 4 \mathrm{~mm})$. To take these data into a common reference space, three registrations were calculated within the Yale BioImage Suite software package (http:// www.bioimagesuite.org/; Duncan et al, 2004). The first registration performed a linear registration between the individual subject's raw functional image and that subject's $2 \mathrm{D}$ anatomical image. The $2 \mathrm{D}$ anatomical image was then linearly registered to the individual's $3 \mathrm{D}$ anatomical image. The $3 \mathrm{D}$ differs from the $2 \mathrm{D}$ in that it has a $1 \times 1 \times 1 \mathrm{~mm}$ resolution, whereas the $2 \mathrm{D} \mathrm{z}$-dimension is set by slicethickness and its $x$-y-dimensions are set by voxel size. Finally, a non-linear registration was computed between the individual 3D anatomical image and a commonly used 3D reference image (the Colin Brain (Holmes et al, 1998) in Montreal Neurological Institute space (Evans et al, 1993)). All three registrations were applied sequentially to the individual normalized $\beta$-maps to bring all data into the common reference space.

Whole-brain AFNI random effects analysis (Cox, 1996 http://afni.nimh.nih.gov) was used to assess condition and group main effects, with subsequent analyses comparing the stress, alcohol-reward cue and neutral-relaxing cue conditions to identify condition-related brain regions and assess $\mathrm{OW} / \mathrm{OB}$ and NW group differences in the three conditions. We applied a $2 \times 3$ ANOVA in which group (OW/OB and NW) and condition (neutral-relaxing/alcohol cues/stress) were treated as within subject fixed effect factors and subject as a random effect factor using the GroupAna program from the AFNI Matlab library (http://afni.nimh. nih.gov/afni/matlab/). Results were masked and converted back into ANALYZE format for viewing in BioImage Suite. The BioimageSuite software employs a built in nonlinear transformation from Montreal Neurological Institute to Talairach coordinates (Lacadie et al, 2008). Data were corrected for multiple comparisons by spatial extent of contiguous supra-thresholded individual voxels at an experiment-wise $p<0.01$. A Monte Carlo simulation as implemented by the Alpha Sim module of the AFNI 
software (Cox, 1996), at a smoothing kernel of $6 \mathrm{~mm}$ and a connection radius of $6.296 \mathrm{~mm}$ on $3.44 \times 3.44 \times 4 \mathrm{~mm}$ voxels was used to determine that an activation volume of 54 original voxels $\left(1467 \mathrm{~mm}^{3}\right)$ satisfied the $p<0.01$ corrected threshold. Finally, to test the a priori hypothesis of significant differences in the VS in the OW/OB $v s$ NW groups, two approaches were used. The first involved visualization of the contrast maps described above, using whole-brain-corrected thresholds. The second involved using small volume correction for the a priori defined region-of-interest (ROI) of VS that was generated from meta-analyses of fMRI investigations of anticipatory reward processing (Figure 3a) (Knutson and Greer, 2008) for which we hypothesized increased activation in the OW/OB group. Small volume correction, done employing a similar method as for whole brain correction analyses, used the Alpha Sim module of the AFNI software. However, instead of applying a whole brain mask, the VS mask in Figure 3a was used. The same parameters were used to determine that an activation volume of 11 original voxels $\left(284 \mathrm{~mm}^{3}\right)$ satisfied the $p<0.01$ corrected threshold. This ROI approach was also used to assess whether activation in the a priori defined VS ROI for reward processing (Knutson and Greer, 2008) was associated with metabolic measures (FPG, insulin, leptin, and HOMA-IR). Pearson correlations were performed with betaweights extracted from the VS ROI.

\section{RESULTS}

\section{Group Demographics}

A total of 48 individuals were included in the study; 21 in the overweight/obese (OW/OB) group and 27 in the NW group. As the groups were defined by OW/OB having a BMI $\geqslant 25 \mathrm{~kg} / \mathrm{m}^{2}$ and $\mathrm{NW}$ having a BMI $18.5-24.9 \mathrm{~kg} / \mathrm{m}^{2}$, they differed in BMI and weight. On average the OW/OB group was overweight, with 14 individuals who were overweight (BMI $25-29.9 \mathrm{~kg} / \mathrm{m}^{2}$ ) and 7 individuals who were obese (BMI $\left.\geqslant 30 \mathrm{~kg} / \mathrm{m}^{2}\right)$. The two groups did not differ with respect to height, race, gender, age, education level, or lifetime prevalence of mood and anxiety disorders (Table 1).

\section{Metabolic Evaluation}

The OW/OB and NW groups differed in measures of FPG, insulin, and leptin. The OW/OB group had a higher mean FPG of $105 \mathrm{mg}$ per $100 \mathrm{ml}$ as compared with the NW group that had a mean FPG of $96.65 \mathrm{mg}$ per $100 \mathrm{ml}$. FPG was found to be $\geqslant 100 \mathrm{mg}$ per $100 \mathrm{ml}$ in $78.6 \%$ of the OW/OB group and $30.4 \%$ of the NW group. The fasting insulin levels in the OW/OB group ranged from 7.0 to $27.7 \mu \mathrm{U} / \mathrm{ml}$, whereas the range in the NW group was from 4.4 to $21.4 \mu \mathrm{U} / \mathrm{ml}$. Having both fasting insulin and glucose measurements available, HOMA-IR was calculated for assessment of insulin resistance in the subject sample. The OW/OB group was also found to have higher mean insulin resistance as calculated by the HOMA-IR (Table 1). Around $71 \%$ of the OB/OW group had HOMA $>2.7$ as compared with $21 \%$ of the NW group. Additionally, leptin levels ranged from 1.0 to $55 \mathrm{ng}$ per $100 \mathrm{ml}$ in the OW/OB group, whereas they ranged from 0.9 to $38 \mathrm{ng}$ per $100 \mathrm{ml}$ in the $\mathrm{NW}$ group.

\section{Subjective Responsiveness of Imagery Task: Anxiety Ratings}

To assess subjective responses to imagery tasks, anxiety rating scores were taken before and after each imagery presentation. In both the $\mathrm{OW} / \mathrm{OB}$ and NW groups, the anxiety rating responses to the scripts as a whole differed, most notably to the stress scripts. Anxiety ratings changed significantly during stress in both groups (Table 1). Overall, the $O W / O B$ group showed greater anxiety with a trend toward higher anxiety during the neutral-relaxing condition. The anxiety ratings tend to differ, but not significantly, to the neutral-relaxing ones (Table 1). There was no statistically significant difference between the anxiety rating scores of the groups at baseline.

Correlations between BMI and metabolic factors. As expected, BMI correlated with FPG, insulin, leptin, and HOMA-IR (Figure 1).

\section{fMRI Results}

Condition main effect. To examine the effect of the experimental manipulation, we assessed condition contrasts between stress, neutral-relaxing, and alcohol cue imagery conditions. (Table 2, Figure 2). Activation differences were observed at $p<0.01$, whole brain corrected, in regions previously implicated in stress and reward responsiveness (for example, within medial frontal cortex and adjacent anterior cingulate gyrus). Similarities between the stress and alcohol cue results reflect shared neurocircuitry underlying appetitive and stress responses (Sinha, 2008).

Group main effect. No brain regions showed a main effect of group that survived whole brain correction at $p<0.05$ (two-tailed). A main effect of group in the VS region survived small volume correction at $p<0.05$ (Figure $3 \mathrm{~b}$ ). $\mathrm{OW} / \mathrm{OB}$ subjects showed greater bilateral VS activation during the neutral-relaxing condition at $p<0.01$, small volume correction (Figure $3 \mathrm{c}$ ) and greater right VS activation during the stress condition at $p<0.05$, small volume correction (Figure 3d). No difference was seen in VS activation during the neutral-relaxing condition as compared with the stress condition in the OW/OB group $(p<0.05)$. Greater VS activity was observed in the stress condition relative to the neutral-relaxing one in the $\mathrm{NW}$ group $(p<0.05)$. The VS activation during the stress condition was not associated with subjective anxiety rating scores in the OW/OB subjects.

Associations between VS activation and metabolic factors. As there was an overall group difference in VS activation, we examined the relationship between metabolic measures and VS activation using the reward-based ROI. In the entire sample, FPG correlated with overall VS activation in the reward-based ROI across conditions (left VS: $r^{2}=0.44$, $p=0.006$; right VS: $r^{2}=0.40, p=0.01$ ) (Figure $4 \mathrm{a}$ and $\mathrm{b}$ ). No other metabolic measures were associated with VS activation across conditions in the entire sample. VS activation in the neutral-relaxing condition, but not in the stress or alcohol-cue conditions, correlated with FPG (left VS: $\left.r^{2}=0.35, p=0.035\right)$. 
Table I Group Demographics and Metabolic Evaluation

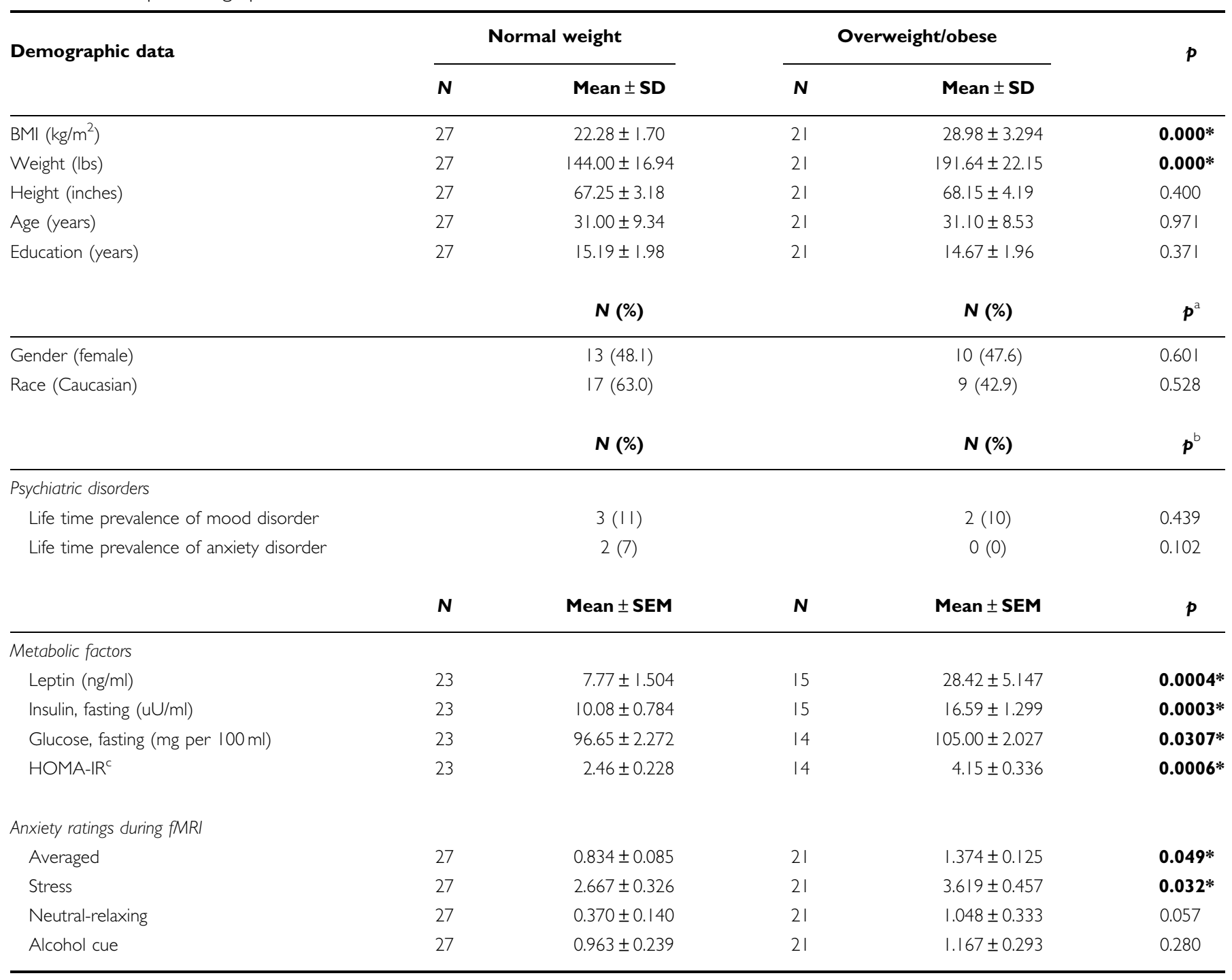

* Statistically significant difference between groups $(p<0.05)$.

Note: Groups were defined on the basis of BMI $\geqslant 25 \mathrm{~kg} / \mathrm{m}^{2}(\mathrm{OW} / \mathrm{OB})$ and $\mathrm{BMI}<18.5-24.9 \mathrm{~kg} / \mathrm{m}^{2}$ (NW). There was a statistically significant difference in the two groups between FPG, insulin, leptin, and HOMA-IR. Outside these measures and the BMI (and related weight) measure used to define the groups, no other statistically significant differences were found between the two groups.

${ }^{\mathrm{a}}$ Fisher ex.

${ }^{b} \chi^{2}-S q$.

${ }^{\mathrm{C}} \mathrm{HOMA}-\mathrm{R}=($ Glucose $(\mathrm{mg}$ per $100 \mathrm{ml}) \times \operatorname{lnsulin}(\mu \mathrm{U} / \mathrm{ml})) \div 405$.

\section{DISCUSSION}

This study revealed that OW/OB individuals, as compared with NW individuals, showed greater VS activation, with the most statistically robust findings implicating the stress and neutral-relaxing conditions. Although no brain regions demonstrated between-group differences surviving whole brain correction, a small volume correction approach identified a significant between-group activation difference in the hypothesized VS region. In addition, the observed VS activation across conditions, and particularly in the neutralrelaxing condition, correlated with FPG. These findings support the hypothesis that during both stress exposure and relaxation $\mathrm{OW} / \mathrm{OB}$ individuals have relatively greater activation of the VS, a region previously implicated in reward processing and stress. Although the finding across conditions suggests that the results may not be specific to condition, the subsequent analyses within each condition suggest that the most statistically robust effects are observed in the neutral-relaxing condition. Furthermore, VS activation correlated with individuals' FPG, suggesting a relationship between metabolic factors and mesolimbic function.

\section{Subjective Responses}

At baseline, there was no statistically significant difference between anxiety rating scores of the groups and the groups did not differ on lifetime frequencies of mood or anxiety 

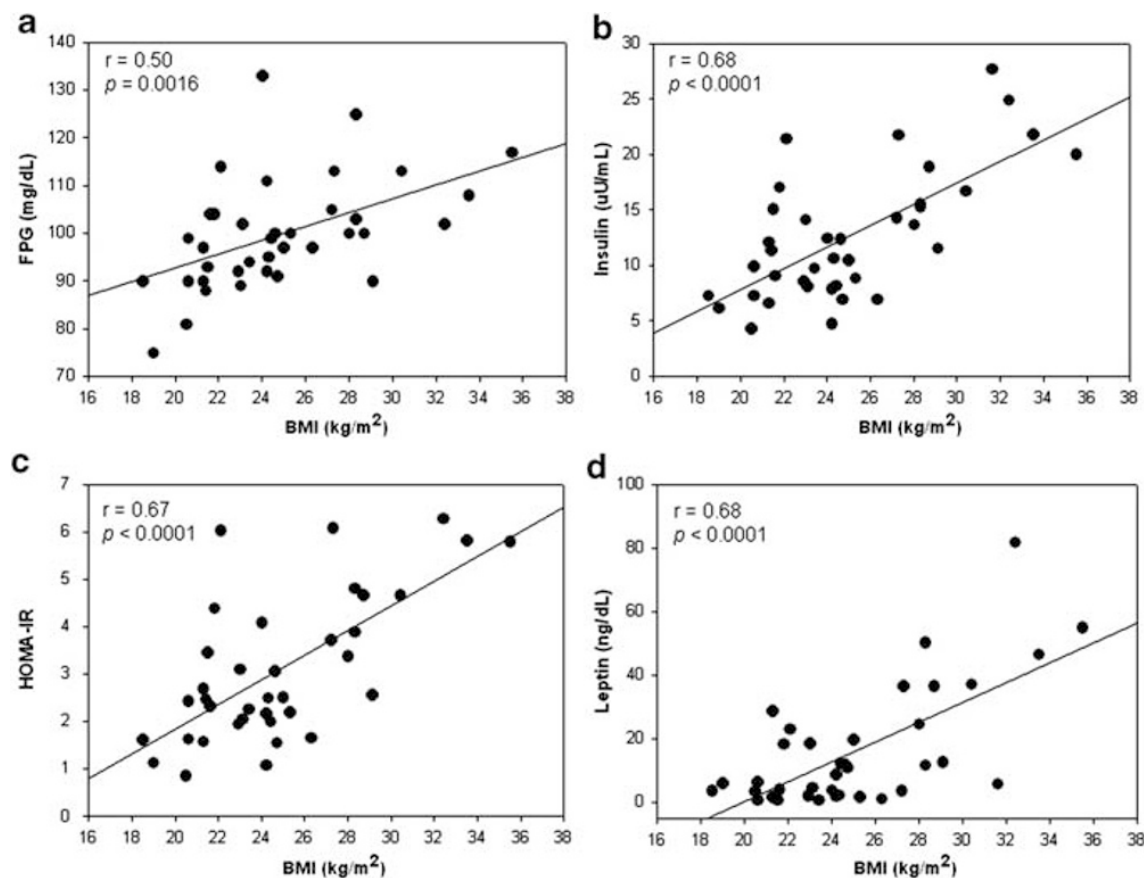

Figure I Pearson correlation of BMI with metabolic factors. As anticipated, BMI correlated significantly with (a) FPG, (b) insulin, (c) HOMA-IR, and (d) leptin.

Table 2 Regional Brain Activation at Threshold $p<0.01$, Whole Brain Corrected, in Stress vs Neutral-relaxing and Alcohol cue vs Neutralrelaxing Conditions

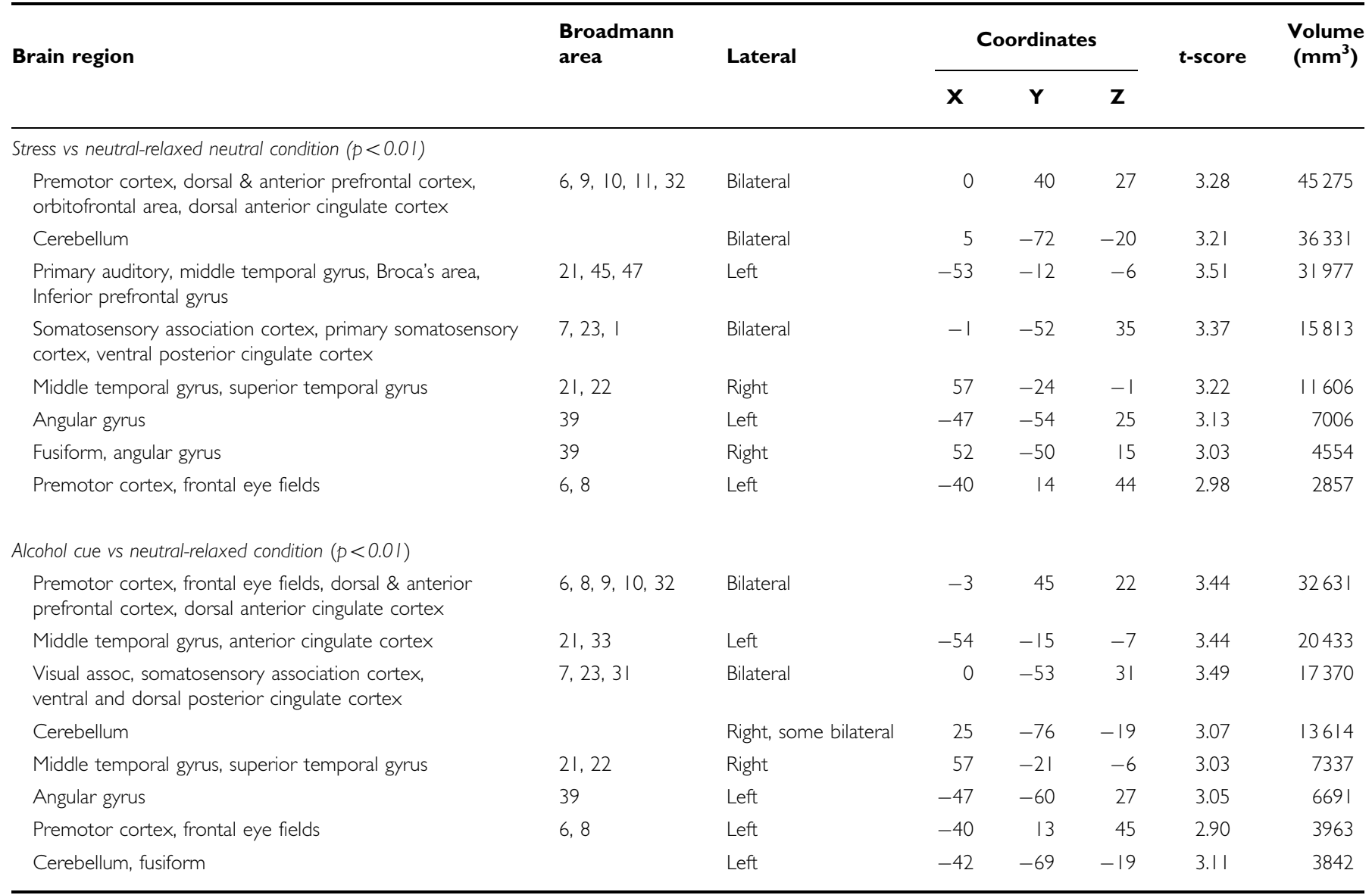

Talairach coordiates used.

Neuropsychopharmacology 


\section{Stress vs. Neutral Alcohol cue vs. Neutral $(\mathrm{p}<0.01)$ $(p<0.01)$}

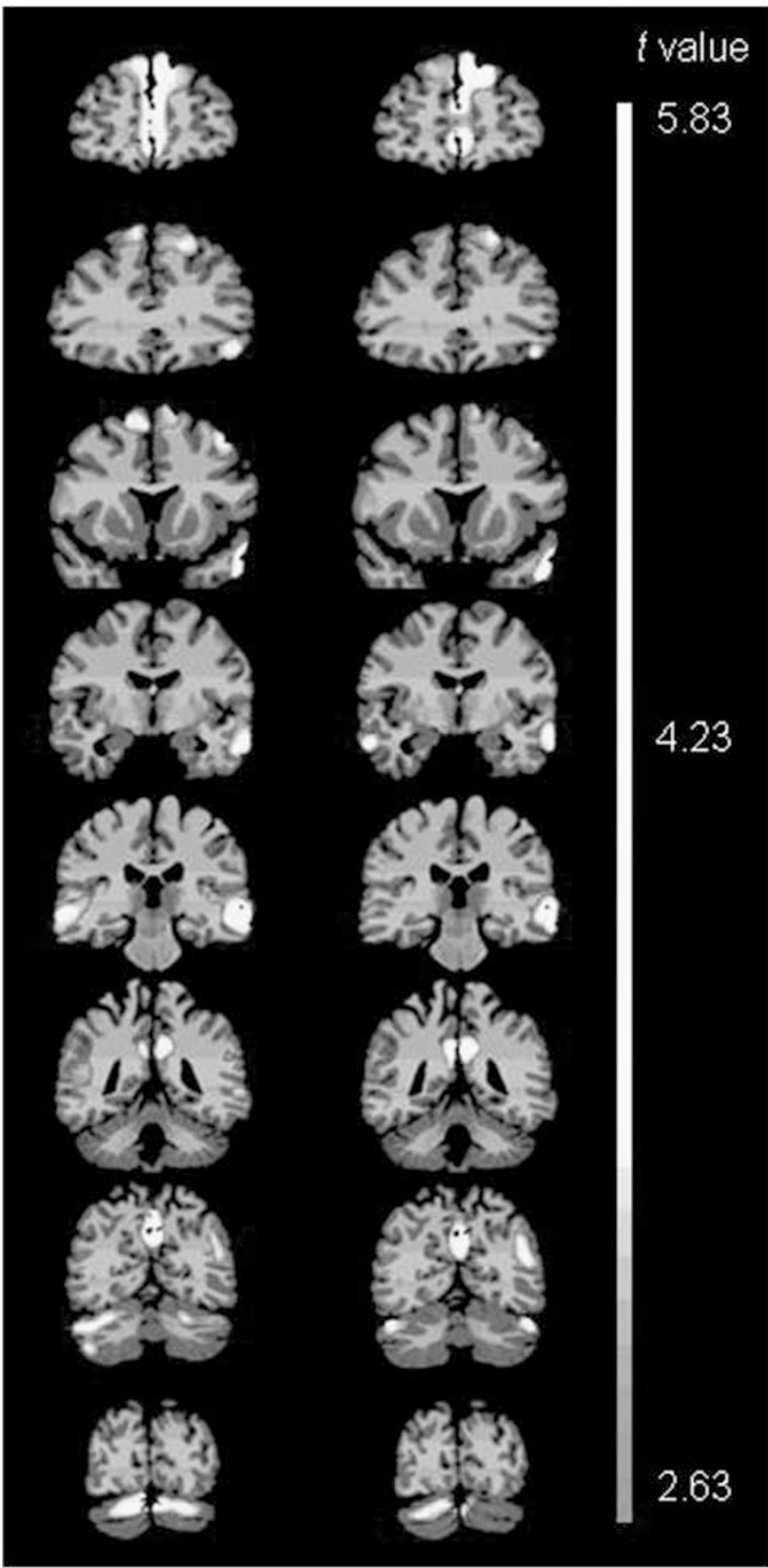

Figure 2 Regional brain activation in all subjects (whole brain corrected at voxel-level threshold $p<0.01$ ) in stress vs neutral-relaxing and alcohol cue vs neutral-relaxing conditions.

disorders. As compared with the NW group, the OW/OB group had higher anxiety responses to the scripts, particularly during the stress condition (Table 1). In the neutral-relaxing condition, the OW/OB individuals tend to have marginally increased anxiety rating scores as compared with the NW individuals, perhaps reflecting an allostatic shift in OW/OB individuals that makes them less able to relax when guided to relax using their personalized most relaxing scenario. The higher subjective anxiety in the OW/OB group may contribute to altered non-homeostatic feeding, such as emotional and stress-induced eating,

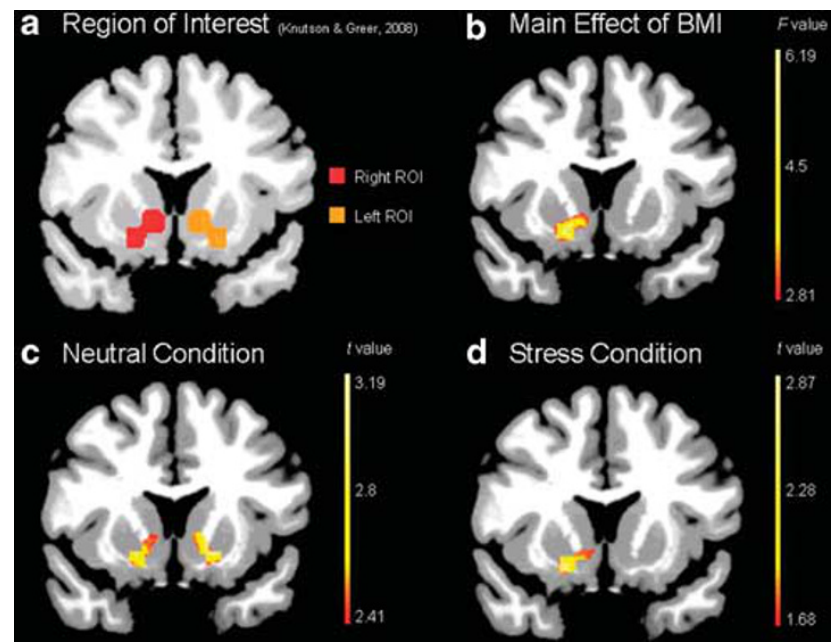

Figure 3 Ventral Striatal brain activation in (a) a priori rewardanticipation region-of-interest (b) main effect of $\mathrm{BMI}(p<0.05$, small volume corrected), (c) neutral-relaxing ( $p<0.01$, small volume corrected) and (d) stress condition ( $p<0.05$, small volume corrected). All coronal slices shown at $y=8$ in Talairach space.

associated with higher BMI (Epel et al, 2004). In that anxiety disorders have been associated with both OW and OB BMIs (Desai et al, 2009), existing data suggest that anxiety is related to BMI at both syndromal and subsyndromal levels.

\section{Brain Activation Differences}

The finding that the OW/OB group demonstrated more robust VS activation than the NW group during relaxation is important because the VS, and specifically the nucleus accumbens, significantly contributes to food reward and anticipation (Kelley et al, 2005; von Deneen et al, 2009). Our findings, therefore, suggest that $\mathrm{OW} / \mathrm{OB}$ individuals as compared with their NW counterparts may have altered reward sensitivity most strongly identifiable in the neutralrelaxing condition and may, therefore, differ in the way that they are motivated to seek or anticipate food. Increased VS activation may influence motivation and reinforcing behaviors for seeking various foods, amounts of food eaten, and pleasure or reward attained from eating food. As a critical reward/motivation region of the brain, the VS, when dysregulated, may promote maladaptive eating behaviors. Consistently, and perhaps supportive of our findings, are observations that morbidly obese individuals exhibit decreased DA D2 receptor availability in the striatum, a finding similar to those seen in addicted individuals (Wang et al, 2001, 2009).

Between-group differences in VS activation were also observed most significantly during the neutral-relaxing condition, and additionally, albeit less so, during the stress condition. The difference seen in the neutral-relaxing condition suggests that $\mathrm{OW} / \mathrm{OB}$ individuals may not be able to modulate VS activation, as well as their NW counterparts, when attempting to achieve a relaxed state. Although the precise reasons for these findings are not completely understood at the present time, several possibilities for these results exist. First, the findings may reflect a 

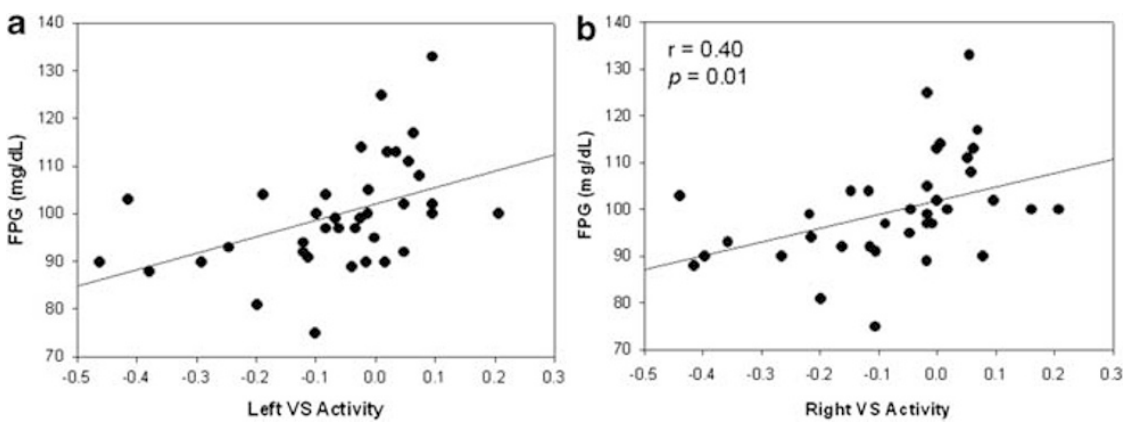

Figure 4 Pearson correlation with FPG and VS activation. (a) Left VS activity and (b) right VS activity and FPG in averaged conditions.

general shift in stress responsiveness. That is, when exposed to stress or trying to achieve a relaxed (or non-stressed) state, OW/OB individuals showed relatively increased VS activation compared with NW individuals that may be due to an allostatic shift in neural functions relating to stressed and non-stressed states (Koob and Le Moal, 2008). Second, a non-mutually exclusive possibility is that several factors (for example, related to metabolism) may similarly influence stressed and relaxed states. The cross-sectional nature of this study does not allow one to discern whether this activation difference precedes the development of a higher BMI or whether a higher BMI state promotes VS activation.

There are several possibilities to consider regarding the finding of changes in VS activation in the OB/OW group in the neutral-relaxing condition. Allostasis, or 'the active process by which the body responds to daily events and maintains homeostasis' may be altered and result in pathophysiology if an individual is chronically exposed to stimuli that increase their allostatic load (McEwen, 2007). The vulnerable individual, perhaps chronically exposed to an excess of highly rewarding, sweet, high-fat foods, may eventually develop maladaptive responsivity in reward/motivation pathways due to increased allostatic load. Alternatively, individual differences linked to striatal function, such as genetic differences in dopamine-related genes, may also predispose certain individuals to weight gain (Stice et al, 2008). Arguably most likely, genetic and environmental factors, such as food exposure interact in obesity, as in other health conditions (Caspi et al, 2003). Furthermore, epidemiological studies indicate elevated odds of anxiety disorders with obesity, particularly among women (Desai et al, 2009). Thus, the ability to relax may be especially relevant to obese women, and direct examination of sex differences in VS activation in larger samples is warranted. Psychosocial stress is associated with higher BMI (Adam and Epel, 2007; Block, 2009), and may contribute to increased vagal tone, increased blood pressure and diminished heart rate variability in obese individuals (Molfino et al, 2009). Factors such as hypertension, insulin resistance, increased waist circumference, and hyperlipidemia indicate an altered metabolic state and classify metabolic syndrome. It is possible that changes related to metabolic makeup alter the basal state of brain reward/ motivational regions as reflected in responses to the neutral-relaxing condition in OW/OB individuals. That is, VS activation may be associated with an individual's chronic metabolic environment, in which chronic changes in metabolic homeostasis subsequently influence appetitive and reward centers in the brain.

\section{Metabolic Measures}

Specific hormonal signals and metabolic factors regulate energy homeostasis through peripheral and central actions. In the setting of increased allostatic load, such as obesity, these hormonal and metabolic factors may become dysregulated and perpetuate maladaptive physiology and behavior (Gao and Horvath, 2007). The stress response may be influenced by neuroendocrine factors, such as insulin and leptin (Adam and Epel, 2007). Our data show that FPG, insulin, and leptin were significantly higher in the OW/OB group relative to NW group. Consistent with the concept of increased metabolic load in obesity, each of these biochemical markers correlated with BMI. Interestingly, the levels of FPG also correlated with VS activation during all three conditions combined, particularly during the neutralrelaxing one. The increased VS activation in the OW/OB group may be related to FPG, but in this study we did not see an association with insulin resistance. Other metabolic measures did not show a significant correlation with VS activity.

Glucose has an important role as a central and peripheral contributor to metabolic status and should be discussed in context with insulin and leptin. Glucose-sensing neurons are found in the ventromedial, lateral hypothalamus, and brain stem (Levin et al, 2004). Hypothalamic input to cortico-striatal circuits has been implicated in providing homeostatic information to motivational neurocircuitry (Chambers et al, 2003), suggesting that the relationship between VS activation and glucose may be mediated in part through hypothalamic function. Leptin and insulin may also influence reward/motivation seeking responses to food. In rats, food-deprivation-induced heroin self administration was diminished by leptin administration into the central nervous system (Shavel et al, 2001). Notably, insulin receptors are located in the hypothalamus (Schwartz et al, 1992), and insulin and leptin receptors are expressed on the dopamine neurons of the ventral tegmental area and the substantia nigra (Figlewicz et al, 2003). Alterations in insulin sensitivity in the ventral tegmental area may alter downstream responses of projections from the ventral tegmental area to the VS (Sandoval et al, 2008). Together, these findings suggest that VS activation differences seen in OW/OB participants may reflect central insulin and leptin resistance and altered glucose utilization, with several 
possible non-mutually exclusive mechanisms that warrant additional investigation.

\section{Stress}

We also found a between-group difference in VS activation during the stress condition. Stress may influence metabolic factors, such as leptin and insulin, contributing to dysregulated homeostatic energy balance and the development of obesity. Stress can elevate glucocorticoid levels (Dallman et al, 2003), and this, in turn, is associated with an increase in glucose levels due to increased gluconeogenesis (Khani and Tayek, 2001), with long-term exposure possibly leading to allostatic changes in insulin resistance and glucose control. Stress also influences reward/motivation pathways and increases vulnerability to addictive behaviors (Sinha, 2008). Altered sensitivity in reward/motivation pathways could increase craving for certain types of foods high in carbohydrates and fats (Dallman et al, 2003), the intake of which could contribute to weight gain. Consistently, psychosocial stress has been associated with increases in BMI (Block, 2009).

Because stress alters natural reward/motivation pathways, it may also be associated with changes in eating behavior and energy homeostasis. The increased VS activation in the OW/OB individuals in the stress and neutral condition and the association between VS activation and hormonal/ metabolic factors suggest a close link between motivation or reward-related neutral circuits and obesity. BMI-grouprelated differences in VS activation during the neutralrelaxing condition suggest that altered striatal function, particularly during stress or while attempting to relax, may contribute to non-homeostatic feeding mechanisms in individuals with high BMI. Also interesting to consider is the individuals we studied were on average overweight (mean BMI $28.98 \mathrm{~kg} / \mathrm{m}^{2}$ ) and not obese. The extent to which these findings extend to, or are magnified in, individuals with obesity requires additional investigation. Interventions to reduce stress may be beneficial in reducing dysfunctional feeding behaviors.

\section{Limitations and Strengths}

There were several limitations to our study. First, this was a preliminary study in which obese individuals were not targeted for recruitment, per se. Although the study included a relatively large sample for neuroimaging, the number of individuals in the obese category (BMI $\geqslant 30 \mathrm{~kg} / \mathrm{m}^{2}$ ) was small, seven in all, and hence the OW and OB groups were combined, thus perhaps 'diluting' effects related to obesity. Second, all study subjects were social drinkers who consumed less than 7 drinks per week. Thus, the extent to which the findings apply to abstainers and individuals who consume more alcohol is not known; however, the sample studied allowed for the use of an appetitive condition (alcohol cue) salient to the entire group. As the two groups were not different in levels of alcohol intake and both groups were light social drinkers, it is not surprising that we found no between-group differences in subjective responses in anxiety or alcohol craving and brain response to alcohol cues. However, responses to food cues are likely more relevant to obesity and need to be examined in future studies. The extent to which food or alcohol cues are rewarding to individuals stratified by BMI status also warrants direct investigation in future studies. An additional limitation was that a biochemical evaluation was not conducted on the day of the fMRI scanning sessions and metabolic factors may vary across days. Additionally, HOMA-IR provides only a rudimentary measure of insulin resistance. Thus, future studies could benefit from measuring metabolic factors in closer proximity to the imaging sessions to more fully assess participants' metabolic profiles. Additionally, a bidirectional relationship may exist between BMI and stress. Stress could lead to increase BMI and increased BMI could lead to increase stress responsivity. Longitudinal, rather than cross-sectional, studies seem best suited to address such questions.

\section{Conclusions}

This preliminary study is the first to demonstrate altered brain activation during stress and neutral-relaxing conditions in OW/OB individuals as compared to NW individuals. The findings of increased VS activation in OW/OB individuals and the correlation of VS activation with FPG are consistent with the hypothesis that $\mathrm{OW} / \mathrm{OB}$ individuals have increased responses in motivation/reward neurocircuitry that are related to peripheral metabolic factors linked to non-homeostatic feeding in individuals with elevated BMIs. The finding of altered VS activation in the neutralrelaxing and stress conditions suggests that treatments targeting stress management or anxiety reductions may prove to be beneficial through their influences on central motivation/reward neural pathways. Future studies are needed to validate and further explore these findings and the role of the neuroendocrine system in modulating the reward system especially in the setting of stress and stressinduced feeding.

\section{ACKNOWLEDGEMENTS}

This work was supported by NIDDK/NIH T32 DK 07058 'Diabetes Mellitus and Disorders of Metabolism', T32 DK 063703-07 'Training in Pediatric Endocrinology and Diabetes Research', the NIH grants R01-AA013892, RL1AA017539, UL1-DE019586, UL1-RR024139 and the PL1-DA024859 and the NIH Roadmap for Medical Research Common Fund.

\section{DISCLOSURE}

The authors declare that Ania M Jastreboff has provided clinical care for research volunteers in the Pfizer Clinical Research Unit in New Haven Connecticut. Marc N Potenza has received financial support or compensation for the following: Dr Potenza consults for and is an advisor to Boehringer Ingelheim; has consulted for and has financial interests in Somaxon; has received research support from the National Institutes of Health, Veteran's Administration, Mohegan Sun Casino, the National Center for Responsible Gaming and its affiliated Institute for Research on Gambling Disorders, and Forest Laboratories, OrthoMcNeil, Oy-Control/Biotie and Glaxo-SmithKline pharmaceuticals; has participated in surveys, mailings or telephone 
consultations related to drug addiction, impulse control disorders or other health topics; has consulted for law offices and the federal public defender's office in issues related to impulse control disorders; provides clinical care in the Connecticut Department of Mental Health and Addiction Services Problem Gambling Services Program; has performed grant reviews for the National Institutes of Health and other agencies; has guest-edited journal sections; has given academic lectures in grand rounds, CME events and other clinical or scientific venues; and has generated books or book chapters for publishers of mental health texts. Cheryl Lacadie: has nothing to disclose. Kwangik A. Hong: has nothing to disclose. Robert S. Sherwin: The authors declare that over the past three years Robert Sherwin has received compensation from Amylin, Biodel, Boehringer Ingelheim, Johnson \& Johnson, Novartis, Mannkind, McKinsey \& Company, Merck, Medtronic, Lily, and Ono Pharmacy. Rajita Sinha: The authors declare that Rajita Sinha is on the Scientific Advisory Board for Embera Neurotherapeutics and is also a consultant for Glaxo-Smith Kline, Pharmaceuticals. The authors report that they have no financial conflicts of interest with respect to the content of this paper.

\section{REFERENCES}

Adam TC, Epel ES (2007). Stress, eating and the reward system. Physiol Behav 91: 449-458.

Block JP (2009). Psychosocial Stress and Change in Weight Among US Adults. American Journal of Epidemiology 170: 181-192.

Caspi A, Sugden K, Moffitt TE, Taylor A, Craig IW, Harrington HL et al (2003). Influence of life stress on depression: moderation by polymorphism in the 5-HTT gene. Science 301: 386-389.

Chambers RA, Taylor JR, Potenza MN (2003). Developmental neurocircuitry of motivation in Adolescents: A critical period of addiction vulnerability. Am J Psychiatry 160: 1041-1052.

Cox RW (1996). AFNI: software for analysis and visualization of functional magnetic resonance neuroimages. Comput Biomed Res 29: 162-173.

Dallman MF, Pecoraro N, Akana SF, la Fleur SE, Gomez F, Houshyar $\mathrm{H}$ et al (2003). Chronic stress and obesity: A new view of 'comfort food'. PNAS 100: 11696-11701.

Desai RA, Manley M, Desai MM, Potenza MN (2009). Gender differences in the association between body mass index and psychopathology. CNS Spectr 14: 372-383.

Duncan JS, Papademetris X, Yang J, Jackowski M, Zeng X, Staib LH (2004). Geometric Strategies for Neuroanatomic Analysis from MRI. NeuroImage 23: 34-45.

Epel E, Jimenez S, Brownell K, Stroud L, Stoney C, Niaura R (2004). Are stress eaters at risk for the metabolic syndrome? Ann NY Acad Sci 1032: 208-210.

Evans AC, Collins DL, Mills SR, Brown ED, Kelly RL, Peters TM (1993). 3D statistical neuroanatomical models from 305 MRI volumes, Proc. IEEE Nucl Sci Symp Med Imaging Conf 95: 1813-1817.

Figlewicz DP, Evans SB, Murphy J, Hoen M, Myers M, Baskin DG (2003). Expression of Receptors for Insulin and Leptin in the Ventral Tegmental Area/Substantia Nigra of the Rat. Brain Research 964: 107-115.

Finkelstein EA, Fieblkorn IC, Wang G (2003). National Medical Spending Attributable to Overweight and Obesity: How Much, And Who's Paying? Health Affairs (Suppl Web Exclusives): W3: 219-226.

First MB, Spitzer RL, Gibbon M, Williams JB (1995). Structured Clinical Interview for DSM-IV Axis I Disorders-Non-Patient
Edition (SCID-I/NP), version 2.0. New York State Psychiatric Institute, Biometrics Research: New York.

Flegal KM, Carroll MD, Ogden CL, Curtin LR (2010). Prevalence and trends in obesity among US adults, 1999-2008. JAMA 303: 235-241.

Frascella J, Potenza MN, Brown LL, Childress AR (2010). Shared brain vulnerabilities open the way for nonsubstance addictions: carving addiction at a new joint? Ann NY Acad Sci 1187: 294-315.

Gao Q, Horvath TL (2007). Neurobiology of feeding and energy expenditure. Annu Rev Neurosci 30: 367-398.

Hill JO, Peters JC (1998). Environmental contributions to the Obesity Epidemic. Science 280: 1371-1374.

Holmes CJ, Hoge R, Collins L, Woods R, Toga AW, Evans AC (1998). Enhancement of MR images using registration for signal averaging. J Comput Assist Tomogr 22: 324-333.

Kelley AE, Berridge KC (2002). The Neuroscience of Natural Rewards: Relevance to Addictive Drugs. J Neurosci 22: 3306-3311.

Kelley AE, Blado BA, Pratt WE, Will MJ (2005). Corticostriatalhypothalamic circuitry and food motivation: Integration of energy, action and reward. Physiol Behav 86: 773-795.

Khani S, Tayek JA (2001). Cortisol increases gluconeogenesis in humans: its role in the metabolic syndrome. Clinical Science 101: 739-747.

Knutson B, Greer SM (2008). Anticipatory affect: neural correlates and consequences for choice. Philos Trans R Soc Lond, B, Biol Sci 363: 3771-3786.

Koob GF, Le Moal M (2008). Addiction and the Brain AntiReward System. Annu Rev Psychol 59: 29-53.

Lacadie CM, Fulbright RK, Rajeevan N, Constable RT, Papademetris X (2008). More accurate Talairach coordinates for neuroimaging using non-linear registration. Neuroimage 42: 717-725.

Levin BE, Routh VH, Kang L, Sanders N, Dunn-Meynell AA (2004). Neuronal Glucosensing What Do We Know After 50 Years? Diabetes 53: 2521-2528.

Li C-S, Kosten TR, Sinha R (2005). Sex differences in brain response to stress imagery in abstinent cocaine dependent individuals - an fMRI study. Biol Psychiatry 57: 487-494.

Miller GA, Levin DN, Kozak MJ, Cook III EW, McLean Jr A, Lang PJ (1987). Individual differences in imagery and the psychophysiology of emotion. Cognition and Emotion 1: 367-390.

McEwen B (2007). Physiology and Neurobiology of Stress and Adaptation: Central Role of the Brain. Physiol Rev 87: 873-904.

Molfino A, Fiorentini A, Tubani L, Martuscelli M, Rossi Fanelli F, Laviano A (2009). Body mass index is related to autonomic nervous system activity as measured by heart rate variability. Eur J Clin Nutr 63: 1263-1265.

Nolfe E, Voet T, Jacobs F, Dierckx R, Lemahieu I (2003). XMedCon- An open-source medical image conversion toolkit. Eur J Nucl Med 30(Supp.2): S246.

Ogden CL, Carroll MD, McDowell MA, Flegal KM (2007). Obesity among adults in the United States - no change since 2003-2004. NCHS data brief no 1. National Center for Health Statistics: Hyattsville, MD.

Pruessner JC, Champagne F, Meaney MJ, Dagher A (2004). Dopamine Release in Response to a Psychological Stress in Humans and Its Relationship to Early Life Maternal Care: A Positron Emission Tomography Study Using [11C]Raclopride. J Neurosci 24: 2825-2831.

Sandoval DA, Cota D, Seeley RJ (2008). The integrative role of CNS fuel-sensing mechanisms in energy balance and glucose regulation. Annu Rev Physiol 70: 513-535.

Schwartz MW, Figlewicz DP, Baskin DG, Woods SC, Porte D (1992). Insulin in the Brain: A Hormonal Regulator of Energy Balance. Endocr Rev 13: 387-414.

Shavel U, Yap J, Shaham Y (2001). Leptin Attenuates Acute Food Deprivation-Induced Relapse to Heroin Seeking. J Neurosci 21: RC129. 


\section{Striatal response and BMI}

AM Jastreboff et al

Sinha R (2001). How Does Stress Increase the Risk of Drug Abuse and Relapse? Psychopharmacology 158: 343-359.

Sinha R (2008). Chronic stress, drug use and vulnerability to addiction. Annals of the New York Academy of Sciences: Addiction Reviews 1141: 105-130.

Sinha R (2009). Modeling stress and drug craving in the laboratory: Implications for addiction treatment development. Addict Biol 14: 84-98.

Sinha R, Fox HC, Hong KA, Bergquist K, Bhagwagar Z, Siedlarz KM (2009). Enhanced Negative Emotion and Alcohol Craving, and Altered Physiological Responses Following Stress and Cue Exposure in Alcohol Dependent Individuals. Neuropsychopharmacology 34: 1198-1208.

Sinha R, Lacadie C, Skudlarski P, Wexler BE (2004). Neural circuits underlying emotional distress in humans. Ann NY Acad Sci 1032: 254-257.

Sinha R, Lacadie C, Skudlarski P, Fulbright RK, Kosten TR, Rounsaville BJ et al (2005). Neural activity associated with stress-induced cocaine craving: An fMRI study. Psychopharmacology 183: 171-180.

Sinha R, Lovallo WR, Parsons OA (1992). Cardiovascular differentiation of emotions. Psychosom Med 54: 422-435.

Sinha R, Talih M, Malison R, Cooney N, Anderson GM, Kreek MJ (2003). Hypothalamic-pituitary-adrenal axis and sympatho-adreno- medullary responses during stress-induced and drug cue-induced cocaine craving states. Psychopharmacology 170: 62-72.

Stice E, Spoor S, Bohon C, Small DM (2008). Response to Food Is Moderated by TaqlA Allele. Science 322: 449-452.

Volkow ND, Wang G-J, Fowler JS, Telang F (2008). Overlapping neural circuits in addiction and obesity: evidence of systems pathology. Philos Trans $R$ Soc 363: 3191-3200.

Volkow ND, Wise RA (2005). How can drug addiction help us understand obesity? Nat Neurosci 8: 555-560.

Von Deneen KM, Gold MS, Liu Y (2009). Food Addiction and Cues in Prader-Willi Syndrome. J Addict Med 3: 19-25.

Wang G-J, Volkow ND, Logan J, Pappas NR, Wong CT, Zhu W et al (2001). Brain dopamine and obesity. Lancet 357: 354-357.

Wang G-J, Volkow ND, Thanos PK, Fowler JS (2004). Similarity Between Obesity and Drug Addiction as Assessed by Neurofunctional Imaging: A Concept Review. J Addict Dis 23: 39-53.

Wang GJ, Volkow ND, Thanos PK, Fowler JS (2009). Imaging of brain dopamine pathways: implications for understanding obesity. J Addict Med 3: 8-18.

Woods SC, Seeley RJ (2000). Adiposity signals and the control of energy homeostasis. Ingestive Behavior and Obesity 16: 894-902.

Supplementary Information accompanies the paper on the Neuropsychopharmacology website (http://www.nature.com/npp) 